\title{
On commuting probabilities in finite groups and rings
}

Research Article

\author{
Martin Juráš, Mihail Ursul
}

\begin{abstract}
We show that the set of all commuting probabilities in finite rings is a subset of the set of all commuting probabilities in finite nilpotent groups of class $\leq 2$. These two sets are equal when restricted to groups and rings with odd number of elements.
\end{abstract}

2010 MSC: 16U80, 05C25, 20P05, 16N40, 20D15

Keywords: Finite group, Finite ring, Commuting probability, Annihilating probability, Nilpotent group, Nilpotent ring

\section{Introduction and preliminaries}

In 1940, Philip Hall [17] introduced the notion of the commuting probability in groups. Feit and Fine [12], derived a combinatorial formula and a generating function for commuting probability in matrix rings over finite fields. In the second half of 1960's, the series of papers [8], [9], [10], [11] by Erdös and Turán, gave birth to the statistical group theory. In the fourth paper, among other results, the authors derived a lower bound for commuting probability in a finite group of order $n$, and showed that the commuting probability in the symmetric group $S_{n}$ is asymptotically equal to $\frac{1}{n}$.

A number of research and expository papers on commuting probability in groups appeared during late sixties and the seventies: Joseph [19], [20], Galagher [13], Gustafson [16], Machale [22], and Rusin [27], to name a few ${ }^{1}$. Rusin [27], characterized all finite groups with commuting probability $>\frac{11}{32}$.

There has also been interest in the study of commuting probability of other algebraic structures, [20]. MacHale [23], investigated the notion of commuting probability in rings. In 1995 Lescot [21], rederived classification of groups with commuting probability $>\frac{1}{2}$, using the notion of isoclinism in groups introduced by Hall [17]. Recently, the commuting probability in semigroups has been studied in [14], [24], [26] and [29].

\footnotetext{
Martin Juráš (Corresponding Author); SCAD, Savannah, GA 31401, USA (email: mjuras@scad.edu, martinjuras@gmail.com).

Mihail Ursul; Department of Mathematics, PNG University of Technology, Lae, PNG (email: mihail.ursul@ gmail.com).

${ }_{1}$ Dixon, provides an extensive list of publications on statistical group theory in the references of his paper [7], up to the year 2002.
} 
Since the dawn of the twenty-first century we have seen an escalation of interest in the study of the commuting probability in groups, and commuting and other types of probabilities in rings, such as anticommuting and annihilating probability. Publications [28], [7], [15], [6] and [18] deal with commuting probability in groups. In papers [3] and [1], Buckley et. al. classified all rings with commuting probability $\geq \frac{11}{32}$ and anticommuting probability $\geq \frac{15}{32}$, respectively.

Throughout this paper, $|A|$ denotes cardinality of the set $A$. $Z(G)$ denotes the center of a group $G$. For $a, b \in G,[a, b]=a^{-1} b^{-1} a b$ denotes the commutator of $a$ and $b$, and $[G, G]$ denotes the derived subgroup of $G$ generated by all commutators in $G$. Recall that $G$ is nilpotent of class $n$, if its lower central series (of normal subgroups) terminate in the trivial subgroup after $n$ steps, i.e.

$$
G=G_{0} \triangleright G_{1} \triangleright \cdots \triangleright G_{n}=\left\{e_{G}\right\},
$$

where $G_{i}=\left[G_{i-1}, G\right]$ for $i=1,2, \ldots, n$, and $G_{n-1} \neq\left\{e_{G}\right\}$. Commuting probability 2 in a group $G$ is defined to be the number

$$
\operatorname{Pr}_{c}(G)=\frac{|\{(a, b) \in G \times G: a b=b a\}|}{|G|^{2}} .
$$

For a class $\mathcal{G}$ of finite groups, the set $\mathfrak{S}_{c}(\mathcal{G})=\left\{\operatorname{Pr}_{c}(G): G \in \mathcal{G}\right\}$ is called the commuting spectrum of $\mathcal{G}$.

Rings are not assumed to be associative or unitary. By $R(+)$ we denote the additive group of $R$.

Recall that a ring $R$ is called antisymmetric if for all $a, b \in R, a b=-b a$. $R$ is called strongly antisymmetric if the dinipotent condition, $a^{2}=0$, is satisfied for all $a \in R$. Strong antisymmetry implies antisymmetry. A ring $R$ is said to be of nilpotent class $\leq n$ if the product of any $n$ elements with any correct distribution of brackets is zero. For a prime $p, R$ is called a $p$-ring if $|R|=p^{n}$ for some positive integer $n$.

The symbol $[\cdot, \cdot]$ denotes the commutator in both a group $G$ and a $\operatorname{ring} R$ (for rings, $[a, b]=a b-b a)$. Whenever needed, we will write $[\cdot, \cdot]_{G}$ and $[\cdot, \cdot]_{R}$ to distinguish between the two cases.

Buckley [2], introduced the following generalization of the notion of commuting probability in rings. Let $f(X, Y)=a X Y+b Y X$ be a formal "non-commutative polynomial" with integer coefficients. For any ring $R$ define a function $f^{R}: R \times R \rightarrow R,(x, y)=a x y+b y x$. Let

$$
\operatorname{Pr}_{f}(R)=\frac{\left|\left\{(x, y) \in R \times R: f^{R}(x, y)=0\right\}\right|}{|R|^{2}} .
$$

For a class $\mathcal{R}$ of finite rings, the set $\mathfrak{S}_{f}(\mathcal{R})=\left\{\operatorname{Pr}_{f}(R): R \in \mathcal{R}\right\}$ is called the $f$-spectrum of $\mathcal{R}$.

Here, we are going to be mostly concerned with the commuting spectrum, $\mathfrak{S}_{c}(\mathcal{R})$ and the annihilating spectrum, $\mathfrak{S}_{a n n}(\mathcal{R})$, with the associated formal "non-commutative polynomials" $f(X, Y)=X Y-Y X$ and $f(X, Y)=X Y$, respectively. The commuting probability and the annihilating probability in a ring $R$ are denoted by $\operatorname{Pr}_{c}(R)$ and $\operatorname{Pr}_{a n n}(R)$, respectively.

We will use the following classes of groups and rings:

$\mathfrak{G}$ the class of finite groups;

$\mathfrak{G}_{\text {nil }}$ the class of finite nilpotent groups;

$\mathfrak{G}_{\text {nil }}^{(2)}$ the class of finite nilpotent groups of class $\leq 2$;

$\mathfrak{R}$ the class of finite rings;

$\mathfrak{R}_{n i l}^{(2)}$ the class of finite nilpotent rings of class $\leq 3$;

2 Some publications use the term commuting degree in place of the commuting probability. 
$\Re_{s a}$ the class of finite strongly antisymmetric rings;

$\mathfrak{R}_{p}$ the class of $p$-rings;

for the class $\mathcal{C}$ of finite sets, denote $\operatorname{ODD}(\mathcal{C})=\{A \in \mathcal{C}:|A|$ is odd $\}$.

Recall the following well know construction. For a given ring $R$, we construct the ring $N(R)$ in the following way: the additive group of $N(R)$ is $(R \times R,+)$ with multiplication $(a, x)(b, y)=(0, a b)$. The following Lemma is immediate.

Lemma 1.1. Let $R$ be a ring. Then $N(R)$ is a nilpotent ring of class at most 3. Furthermore, if $f(X, Y)=a X Y+b Y X$ is a formal non-commutative polynomial with integer coefficients and $R$ is finite, then

$$
\operatorname{Pr}_{f}(R)=\operatorname{Pr}_{f}(N(R))
$$

In particular, the Lemma implies

$$
\mathfrak{S}_{f}(\mathfrak{R})=\mathfrak{S}_{f}\left(\mathfrak{R}_{\text {nil }}^{(2)}\right)
$$

Ever since it was discovered that there are no finite groups with commuting probability in the open interval $\left.\frac{5}{8}\right), 1$ ), there has been an interest to understand the structure of the commuting spectrum of groups, and later, the structure of the commuting spectrum of rings and semigroups. The commuting spectrum for semigroups turned out to be the simplest to understand. Givens [14] showed that the commuting spectrum for semigroups is dense in the interval $[0,1]$. Later Ponomarenko and Selinski [26] proved that for any rational number in $r \in(0,1]$, there is a finite semigroup $S$ such that the commuting probability in $S$ is equal to $r$. Soule [29] found a single family of semigroups that has this property. These semigroups are defined as follows. Let $X=\left\{x_{1}, x_{2}, \ldots, x_{m}\right\} \in\{1,2, \ldots, n\}$ and $x_{1}<x_{2}<x_{3}<\ldots<x_{m}$. Set $x_{0}=0$. Define a semigroup $S(n, X)$ as follows: For any $a, b \in\{1,2, \ldots, n\}$

$$
a \star b= \begin{cases}x_{i} & \text { if } x_{i-1}<a \leq x_{i} \text { and } b \geq x_{m}, \\ \max \{a, b\} & \text { if } a>x_{m} \text { or } b>x_{m} .\end{cases}
$$

The author than shows that any rational commuting probability can be achieved by an appropriate choice of parameters.

Contrastingly, for groups, Hegarty [18] showed that for any limit point ${ }^{3} l$ of $\mathfrak{S}_{c}(\mathfrak{G}), l \in\left(\frac{2}{9}, 1\right]$, there is no increasing sequence of numbers $\left\{a_{n}\right\} \subset \mathfrak{S}_{c}(\mathfrak{G})$, such that $l=\lim _{n \rightarrow \infty} a_{n}$.

Recently, Buckley and MacHale investigated relations between the commuting spectra of finite groups and rings. Comparing the structure of these two spectra for large probabilities, the authors formulated two conjectures, [4], page 9:

Conjecture 1. $\mathfrak{S}_{c}(\mathfrak{R}) \subset \mathfrak{S}_{c}(\mathfrak{G})$.

Conjecture 2. $\mathfrak{S}_{c}(\mathfrak{R})=\mathfrak{S}_{c}\left(\mathfrak{G}_{\text {nil }}\right)$ or $\mathfrak{S}_{c}(\mathfrak{R})=\mathfrak{S}_{c}\left(\mathfrak{G}_{\text {nil }}^{(2)}\right)$.

This paper positively resolves the first conjecture and partially resolves the second one. ${ }^{4}$

\section{Main results}

Theorem 2.1. $\mathfrak{S}_{c}(\mathfrak{R}) \subseteq \mathfrak{S}_{c}\left(\mathfrak{G}_{n i l}^{(2)}\right) \subseteq \mathfrak{S}_{a n n}\left(\mathfrak{R}_{s a} \cap \mathfrak{R}_{\text {nil }}^{(2)}\right)$.

\footnotetext{
${ }^{3} x$ is a limit point of a set $S$ if every neighborhood of $x$ contains at least one point of $S$ different from $x$ itself.

4 The authors would like to thank Victor Bovdi for his interest in this paper.
} 
In [5], the authors determined all values in $\mathfrak{S}_{c}(\mathfrak{R})$ that are $\geq \frac{11}{32}$. These are

$$
1, \frac{7}{16}, \frac{11}{27}, \frac{25}{64}, \frac{11}{32}, \quad \text { and } \quad \frac{2^{2 k}+1}{2^{2 k+1}} \text { for } k=1,2,3, \ldots
$$

Thus, $\frac{1}{2} \notin \mathfrak{S}_{c}(\mathfrak{R})$. But, $\frac{1}{2} \in \mathfrak{S}_{c}(\mathfrak{G}),([27]$, page 246$)$, and so $\mathfrak{S}_{c}(\mathfrak{R}) \neq \mathfrak{S}_{c}(\mathfrak{G})$. In particular, $\operatorname{Pr}_{c}\left(S_{3}\right)=\frac{1}{2}$ (see [20]); $S_{3}$ denotes the symmetric group of order 3. This, together with the first inclusion of Theorem 2.1, positively resolves Conjecture 1. As for Conjecture 2, the Theorem states $\mathfrak{S}_{c}(\mathfrak{R}) \subseteq \mathfrak{S}_{c}\left(\mathfrak{G}_{n i l}^{(2)}\right)$. Now that we know $\mathfrak{S}_{c}(\mathfrak{R})$ is a subset of the potentially smaller one of the two sets, $\mathfrak{S}_{c}\left(\mathfrak{G}_{\text {nil }}^{(2)}\right)$ and $\mathfrak{S}_{c}\left(\mathfrak{G}_{\text {nil }}\right)$ (it is unknown whether or not $\left.\mathfrak{S}_{c}\left(\mathfrak{G}_{\text {nil }}^{(2)}\right)=\mathfrak{S}_{c}\left(\mathfrak{G}_{\text {nil }}\right)\right)$, we ask the following question: Does

$$
\mathfrak{S}_{c}(\mathfrak{R})=\mathfrak{S}_{c}\left(\mathfrak{G}_{\text {nil }}^{(2)}\right)
$$

hold true? We don't know. But, Equation (2) does hold true, when restricted to finite groups and finite rings with odd number of elements. In fact, we prove the following:

\section{Theorem 2.2.}

$$
\mathfrak{S}_{c}(\operatorname{ODD}(\mathfrak{R}))=\mathfrak{S}_{c}\left(\operatorname{ODD}\left(\mathfrak{G}_{n i l}^{(2)}\right)\right)=\mathfrak{S}_{a n n}\left(\operatorname{ODD}\left(\mathfrak{R}_{s a} \cap \mathfrak{R}_{\text {nil }}^{(2)}\right)\right)
$$

Next, we would like to formulate a condition, purely in terms of probabilities in rings, that would imply Equation (2). Using Theorem 2.1, one obvious choice could be $\mathfrak{S}_{\text {ann }}\left(\mathfrak{R}_{s a} \cap \mathfrak{R}_{\text {nil }}^{(2)}\right) \subseteq \mathfrak{S}_{c}(\mathfrak{R})$. We can do slightly better. Because things are working smoothly when restricted to rings with odd number of elements, it is sufficient to focus on the "trouble makers" which are the 2-rings.

Proposition 2.3. If $\mathfrak{S}_{a n n}\left(\mathfrak{R}_{s a} \cap \mathfrak{R}_{\text {nil }}^{(2)} \cap \mathfrak{R}_{2}\right) \subseteq \mathfrak{S}_{c}(\mathfrak{R})$, then Equation (2) holds true.

The condition of Proposition 2.3 implies a stronger statement: If $\mathfrak{S}_{a n n}\left(\mathfrak{R}_{s a} \cap \mathfrak{R}_{n i l}^{(2)} \cap \mathfrak{R}_{2}\right) \subseteq \mathfrak{S}_{c}(\mathfrak{R})$, then both inclusions in Theorem 2.1 can be replaced by equal signs. Note that if there is a counterexample to the condition above, i.e. if there exists a ring $R$ such that $R \in \mathfrak{R}_{s a} \cap \mathfrak{R}_{n i l}^{(2)} \cap \mathfrak{R}_{2}$ and $\operatorname{Pr}_{\text {ann }}(R) \notin \mathfrak{S}_{c}(\mathfrak{R})$, then $\operatorname{Pr}_{a n n}(R)<\frac{11}{32}$. We conjecture that $\mathfrak{S}_{c}(\mathfrak{R})=\mathfrak{S}_{a n n}\left(\mathfrak{R}_{s a}\right)$.

\section{Proofs}

Let $N$ be an associative nilpotent ring of class $n$. Then $N$, endowed with "circular multiplication", $a \circ b=a+b+a b$, is a group which we will denote by $G_{N} \cdot{ }^{5} 0$ is the unit element in $G_{N}$ and $a^{-1}=$ $-a+a^{2}-a^{3}+\cdots+(-1)^{n-1} a^{n-1}$ is the inverse of $a$ in $G_{N}, a \circ a^{-1}=a^{-1} \circ a=0$. Since, $a b=b a$ if and only if $a \circ b=b \circ a$, then, if $N$ is finite,

$$
\operatorname{Pr}_{c}(N)=\operatorname{Pr}_{c}\left(G_{N}\right)
$$

Lemma 3.1. Let $N$ be a nilpotent ring of class at most 3 (hence, also an associative ring). Let $a, b, c \in N$. Then

(i) $[a, b]_{G_{N}}=[a, b]_{N}$,

(ii) $[a, b]_{G_{N}} \circ[c, d]_{G_{N}}=[a, b]_{N}+[c, d]_{N}$,

(iii) $G_{N}$ is a nilpotent group of class $\leq 2$.

\footnotetext{
5 Another way to associate a group to a ring such that their commuting probabilities equate can be obtained by modifying a construction of Mal'cev [25]. For an arbitrary ring $R$, define a binary operation on $R \times R$ by $(a, b) \cdot(c, d)=(a+c, a c+b+d)$. This operation is associative, has unit $(0,0)$ and $(a, b)^{-1}=\left(-a, a^{2}-b\right)$. $G=(R \times R, \cdot)$ is a nilpotent group of class at most 2 and $\operatorname{Pr}_{c}(R)=\operatorname{Pr}_{c}(G)$. Note that, unlike the construction of $G_{N}$, the ring $R$ is not required to be nilpotent or associative!
} 
Proof. (i) follows by direct computation.

(ii). By $(i)$,

$$
\begin{gathered}
{[a, b]_{G_{N}} \circ[c, d]_{G_{N}}=[a, b]_{N} \circ[c, d]_{N}} \\
=[a, b]_{N}+[c, d]_{N}+[a, b]_{N}[c, d]_{N}=[a, b]_{N}+[c, d]_{N} .
\end{gathered}
$$

(iii). By (i), $\left[[a, b]_{G_{N}}, c\right]_{G_{N}}=\left[[a, b]_{N}, c\right]_{N}=0$.

Let $G$ be a nilpotent group of class $\leq 2$ and let $Z=Z(G)$ be the center of $G$. Then $G / Z$ is abelian. By $R_{G}$, denote the ring with the additive group $G / Z \oplus Z$, and the multiplication defined by

$$
(a Z, x) \cdot(b Z, y)=(Z,[a, b]),
$$

where $[a, b]=a^{-1} b^{-1} a b$ is the commutator in $G$. Explicitly, the addition in $R_{G}$ is given by

$$
(a Z, x)+(b Z, y)=(a b Z, x y) .
$$

$\left(Z, e_{G}\right)$ is the zero element and $\left(a^{-1} Z, x^{-1}\right)$ is the additive inverse of $(a Z, x)$.

To verify that $R_{G}$ is indeed a ring, the distributive laws have to be satisfied. Let $a, b, c \in G$ and $x, y, z \in Z$. We have

$$
(c Z, z) \cdot((a Z, x)+(b Z, y))=(c Z, z) \cdot(a b Z, x y)=(Z,[c, a b]) .
$$

On the other hand,

$$
(c Z, z) \cdot(a Z, x)+(c Z, z) \cdot(b Z, y))=(Z,[c, a])+(Z,[c, b])=(Z,[c, a][c, b]) .
$$

Using $[G, G] \subseteq Z$, we deduce

$$
\begin{gathered}
{[c, a][c, b]=c^{-1} a^{-1} c a c^{-1} b^{-1} c b=c^{-1}\left[a, c^{-1}\right] b^{-1} c b=c^{-1} b^{-1}\left[a, c^{-1}\right] c b} \\
=c^{-1} b^{-1} a^{-1} c a c^{-1} c b=c^{-1} b^{-1} a^{-1} c a b=c^{-1}(a b)^{-1} c(a b)=[c, a b] .
\end{gathered}
$$

Hence, the left distributive law is satisfied. The proof of the right distributive law is similar. ${ }^{6}$

Lemma 3.2. Let $G$ be a nilpotent group of class at most 2 . Then $R_{G}$ is a strongly antisymmetric nilpotent ring of class at most 3 . If $G$ is finite, then $\left|R_{G}\right|=|G|$ and

$$
\operatorname{Pr}_{c}(G)=\operatorname{Pr}_{a n n}\left(R_{G}\right) \text {. }
$$

Proof. $\left|R_{G}\right|=|G / Z||Z|=|G| . R_{G}^{3}=0$ and strong antisymmetry of $R_{G}$ follows immediately from the multiplication formula (4). To prove (5), it suffices to note that $(a Z, x) \cdot(b Z, y)=(Z,[a, b])=\left(Z, e_{G}\right)$ if and only if $[a, b]=e_{G}$. But, this is exactly when $a b=b a$.

\section{Proof of Theorem 2.1.}

We first show that $\mathfrak{S}_{c}(\mathfrak{R}) \subseteq \mathfrak{S}_{c}\left(\mathfrak{G}_{n i l}^{(2)}\right)$. Let $r \in \mathfrak{S}_{c}(\mathfrak{R})$ By Lemma 1.1, there is a nilpotent ring $N$ of class at most 3 such that $r=\operatorname{Pr}_{c}(N)$. By Lemma 3.1(iii), $G_{N}$ is a nilpotent group of class at most 2 and by Equation $(3), \operatorname{Pr}_{c}\left(G_{N}\right)=\operatorname{Pr}_{c}(N)$. We conclude that $r \in \mathfrak{S}_{c}\left(\mathfrak{G}_{n i l}^{(2)}\right)$.

To prove the second inclusion, consider $G \in \mathfrak{G}_{n i l}^{(2)}$. By Lemma 3.2 $R_{G} \in \mathfrak{R}_{s a} \cap \mathfrak{R}_{\text {nil }}^{(2)}$ and $\operatorname{Pr}_{a n n}\left(R_{G}\right)=\operatorname{Pr}_{c}(G)$.

\footnotetext{
${ }^{6}$ Proposition 3 [4], states that the condition $[c, a][c, b]=[c, a b]$ for all $a, b, c \in G$ is equivalent to $G$ being nilpotent of class $\leq 2$.
} 
Lemma 3.3. Let $R$ be a finite antisymmetric ring and with odd number of elements. Then

$$
\operatorname{Pr}_{c}(R)=\operatorname{Pr}_{a n n}(R)
$$

Proof. In an antisymmetric ring, $a b=-b a$. Hence, $a b=b a$ iff $2 a b=0$. Since $|R|$ is odd, $2 a b=0$ iff $a b=0$.

\section{Proof of Theorem 2.2.}

To prove $\mathfrak{S}_{c}(\operatorname{ODD}(\mathfrak{R})) \subseteq \mathfrak{S}_{c}\left(\operatorname{ODD}\left(\mathfrak{G}_{n i l}^{(2)}\right)\right) \subseteq \mathfrak{S}_{a n n}\left(\operatorname{ODD}\left(\mathfrak{R}_{s a} \cap \mathfrak{R}_{\text {nil }}^{(2)}\right)\right)$, we follow the proof of Theorem 2.1 and note that $|N|=\left|G_{N}\right|$ and $|G| \stackrel{\text { nil }}{=}\left|R_{G}\right|$.

To conclude the proof of Theorem 2.2, it suffices to show

$$
\mathfrak{S}_{a n n}\left(\operatorname{ODD}\left(\mathfrak{R}_{s a} \cap \mathfrak{R}_{\text {nil }}^{(2)}\right)\right) \subseteq \mathfrak{S}_{c}(\operatorname{ODD}(\mathfrak{R}))
$$

Let $r \in \mathfrak{S}_{a n n}\left(\operatorname{ODD}\left(\mathfrak{R}_{s a} \cap \mathfrak{R}_{\text {nil }}^{(2)}\right)\right)$ and let $R \in \operatorname{ODD}\left(\mathfrak{R}_{s a} \cap \mathfrak{R}_{\text {nil }}^{(2)}\right)$ such that $r=\operatorname{Pr}_{a n n}(R)$. By Lemma 3.3, $r=\operatorname{Pr}_{a n n}(R)=\operatorname{Pr}_{c}(R) \in \mathfrak{S}_{c}(\mathrm{ODD}(\Re))$.

For a noncommutative formal polynomial $f(X, Y)=a X Y+b Y X$ and rings $R_{1}$ and $R_{2}$,

$$
\operatorname{Pr}_{f}\left(R_{1} \times R_{2}\right)=\operatorname{Pr}_{f}\left(R_{1}\right) \operatorname{Pr}_{f}\left(R_{2}\right) .
$$

Let $p$ be a prime number and let $\mathcal{C}$ be a class of finite rings. Denote $\mathcal{C}_{p}=\mathcal{C} \cap \mathfrak{R}_{p}$. Assume $\mathcal{C}$ is closed under cartesian products. Then $\mathcal{C}_{p}$ is closed under cartesian products and both $\mathfrak{S}_{f}(\mathcal{C})$ and $\mathfrak{S}_{f}\left(\mathcal{C}_{p}\right)$ are multiplicative monoids. Furthermore, values in $\mathfrak{S}_{f}(\mathcal{C})$ are finite products of values taken from the set $\bigcup_{p} \mathfrak{S}_{f}\left(\mathcal{C}_{p}\right)$, where $p$ runs over all prime numbers. We say that a class $\mathcal{C}$ of finite rings is hereditary, if any subring of a ring in $\mathcal{C}$ is also in $\mathcal{R}$. If a class $\mathcal{C}$ is hereditary, then $\mathcal{C}_{p}$ is also hereditary.

\section{Proof of Proposition 2.3.}

It is easy to see that the class $\mathcal{C}=\mathfrak{R}_{\text {sa }} \cap \mathfrak{R}_{\text {nil }}^{(2)}$ is hereditary and closed under cartesian products. Assume $\mathfrak{S}_{\text {ann }}\left(\mathcal{C} \cap \mathfrak{R}_{2}\right) \subseteq \mathfrak{S}_{c}(\mathfrak{R})$. By Theorem 2.2, $\mathfrak{S}_{\text {ann }}(\mathrm{ODD}(\mathcal{C}))=\mathfrak{S}_{c}(\mathrm{ODD}(\mathfrak{R}))$ and so $\mathfrak{S}_{\text {ann }}(\mathcal{C} \cap$ $\left.\mathfrak{R}_{p}\right) \subseteq \mathfrak{S}_{c}(\mathfrak{R})$ for a prime $p \neq 2$. Hence, for all primes $p$, the monoids $\mathfrak{S}_{a n n}\left(\mathcal{C} \cap \mathfrak{R}_{p}\right) \subseteq \mathfrak{S}_{c}(\mathfrak{R})$ and so $\mathfrak{S}_{\text {ann }}(\mathcal{C}) \subseteq \mathfrak{S}_{c}(\mathfrak{R})$. By Theorem 2.1, the reverse inclusion is satisfied, thus the proposition follows.

\section{References}

[1] S. M. Buckley, D. MacHale, Y. Zelenyuk, Finite rings with large anticommuting probability, Appl. Math. Inf. Sci. 8(1) (2014) 13-25.

[2] S. M. Buckley, Distributive algebras, isoclinism, and invariant probabilities, Contemp. Math. 634 (2015) 31-52.

[3] S. M. Buckley, D. MacHale, Commuting probability for subrings and quotient rings, J. Algebra Comb. Discrete Appl. 4(2) (2017) 189-196.

[4] S. M. Buckley, D. MacHale, Contrasting the commuting probabilities of groups and rings, preprint.

[5] S. M. Buckley, D. MacHale, Á. N. Shé, Finite rings with many commuting pairs of elements, preprint.

[6] A. K. Das, R. K. Nath, A characterisation of certain finite groups of odd order, Math. Proc. R. Ir. Acad. 111(2) (2011) 67-76.

[7] J. Dixon, Probabilistic group theory, C. R. Math. Acad. Sci. Soc. R. Can. 24(1) (2002) 1-15.

[8] P. Erdös, P. Turán, On some problems of a statistical group-theory. I, Z. Wahrschein. Verw. Gebiete. 4 (1965) 175-186.

[9] P. Erdös, P. Turán, On some problems of a statistical group-theory. II, Acta Math. Acad. Sci. Hungar. 18 (1967) 151-163. 
[10] P. Erdös, P. Turán, On some problems of a statistical group-theory. III, Acta Math. Acad. Sci. Hungar. 18 (1967) 309-320.

[11] P. Erdös, P. Turán, On some problems in statistical group-theory. IV, Acta Math. Acad. Sci. Hungar. 19 (1968) 413-453.

[12] W. Feit, N. J. Fine, Pairs of commuting matrices over a finite field, Duke Math. J. 27(1) (1960) 91-94.

[13] P. X. Gallagher, The number of conjugacy classes in a finite group, Math. Z. 118 (1970) 175-179.

[14] B. Givens, The probability that two semigroup elements commute can be almost anything. College Mathematics Journal 39(5) (2008) 399-400.

[15] R. M. Guralnick, G. R. Robinson, On commuting probability in finite groups, J. Algebra 300(2) (2006) 509-528.

[16] W. H. Gustafson, What is the probability that two group elements commute?, Amer. Math. Monthly 80(9) (1973) 1031-1034.

[17] P. Hall, The classification of prime-power groups, J. Reine. Agnew. Math. 182 (1940) 130-141.

[18] P. Hegarty, Limit points in the range of the commuting probability function on finite groups, J. Group Theory 16 (2013) 235-247.

[19] K. S. Joseph, Commutativity in non-abelian groups, Ph.D. Thesis, University of California, Los Angeles (1969).

[20] K. S. Joseph, Several conjectures on commutativity in algebraic structures, Amer. Math. Monthly 84(7) (1977) 550-551.

[21] P. Lescot, Isoclinism classes and commutativity degrees of finite groups, J. Algebra 177(3) (1995) $847-869$.

[22] D. MacHale, How commutative can a non-commutative group be?, Math. Gaz. 58 (1974) 199-202.

[23] D. MacHale, Commutativity in finite rings, Amer. Math. Monthly 84(1) (1976) 30-32.

[24] D. MacHale, Probability in fnite semigroups, Irish Math. Soc. Bull. 25 (1990) 64-68.

[25] A. I. Mal'cev, On a correspondence between rings and groups, in Fifteen papers on algebra, AMS translation American Mathematical Soc. (1965) 221-232.

[26] V. Ponomarenko, N. Selinski, Two semigroup elements can commute with any positive rational probability, College Math. J. 43(4) (2012) 334-336.

[27] D. J. Rusin, What is the probability that two elements of a finite group commute?, Pacific J. Math. 82(1) (1979) 237-247.

[28] Á. N. Shé, Commutativity and generalizations in finite groups, Ph.D. Thesis, University College Cork (2000).

[29] M. Soule, A single family of semigroups with every positive rational commuting probability, College Math. J. 45(2) (2014) 136-139. 